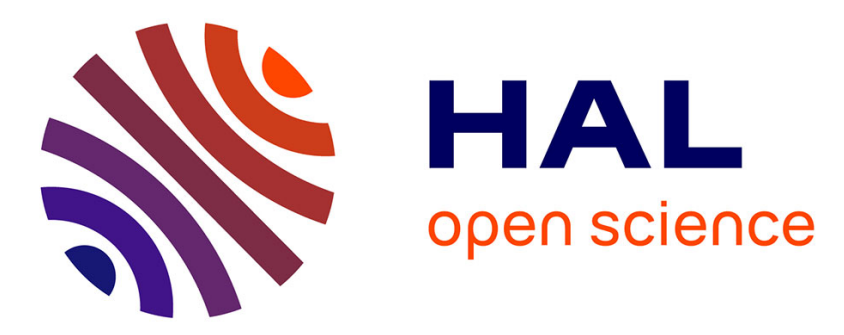

\title{
A note on market completeness with American put options \\ Luciano Campi
}

\section{- To cite this version:}

Luciano Campi. A note on market completeness with American put options. 2011. hal-00566235

\section{HAL Id: hal-00566235 \\ https://hal.science/hal-00566235}

Preprint submitted on 15 Feb 2011

HAL is a multi-disciplinary open access archive for the deposit and dissemination of scientific research documents, whether they are published or not. The documents may come from teaching and research institutions in France or abroad, or from public or private research centers.
L'archive ouverte pluridisciplinaire HAL, est destinée au dépôt et à la diffusion de documents scientifiques de niveau recherche, publiés ou non, émanant des établissements d'enseignement et de recherche français ou étrangers, des laboratoires publics ou privés. 


\title{
A note on market completeness with American put options
}

\author{
Luciano CAMPI*广
}

This version: December, 2010

\begin{abstract}
We consider a non necessarily complete financial market with one bond and one risky asset, whose price process is modelled by a suitably integrable, strictly positive, càdlàg process $S$ over $[0, T]$. Every option price is defined as the conditional expectation under a given equivalent (true) martingale measure $\mathbb{P}$, the same for all options. We show that every positive contingent claim on $S$ can be approximately replicated (in $L^{2}$-sense) by investing dynamically in the underlying and statically in all American put options (of every strike price $k$ and with the same maturity $T)$. We also provide a counter-example to static hedging with European call options of all strike prices and all maturities $t \leq T$.
\end{abstract}

Keywords: market completeness, American put, Tanaka's formula, European call, marginals.

MS Classification (2000): 91B28, 60G40, 60G44, 60G48.

\section{Introduction}

The aim of this paper is to investigate the issue of hedging in a market where agents are allowed to invest continuously on time in a risky asset and statically in American put options of all strike prices and with a fixed maturity $T$. Such additional investment opportunities are of particular interest in incomplete markets, in which case the payoffs cannot, in general, be replicated by a trading strategy in the underlying.

Since in real financial markets many types of options are becoming more and more liquid, it is very natural to reformulate hedging and optimal investment problems incorporating those larger trading opportunities. Indeed, in the recent years many papers have treated problems like absence of arbitrage, hedging, optimal portfolio choice in a financial market where investors are allowed to trade in the underlying assets as well as to assume static positions in some class of derivatives. Here, we recall only few of them: Campi [1] for no-arbitrage and completeness issues, the papers by Ilhan et al. [11, 12] and Carr et al. [5] for optimal investment problems, and the more recent papers by Schweizer and Wissel [20, 21] and by Jacod and Protter [14] where an HJM approach for European call options is developed.

Our paper is much closer in spirit to that part of literature initiated by Ross [19], where it has been shown that in a single period model with a finitely many states and $n$ stocks, simple options

*CEREMADE, Université Paris Dauphine. Place du Maréchal De Lattre de Tassigny, 75775 Paris Cedex 16. E-mail: campi@ceremade.dauphine.fr

${ }^{\dagger}$ I wish to thank Sara Biagini, José M. Corcuera, Jerôme Renault and two anonymous referees for many valuable remarks. I also thank the "Chair Les Particuliers Face aux Risques", Fondation du Risque (Groupama-ENSAEDauphine), and the GIP-ANR "Croyances" project. The usual disclaimer applies. 
(i.e. written on only one stock) can be replicated by trading in European call (or put) options on that stock. Ross [19] established also the existence of a portfolio in all $n$ stocks such that call (or put) options written on that portfolio span the set of all options, simple as well as complex, i.e. written on all stocks at the time. This result has been generalized in an infinite state space setting by Green and Jarrow [10] (see also Nachman [16]). For a similar result in a dynamic discrete-time setting with a general state space, we quote only a paper by Rogge [18], where the market completeness is characterized in terms of a so-called "call-completeness": there exists a strike price $k>0$ such that if a claim is attainable then also a call written on it with strike $k$ is.

A more explicit result has been established in Carr and Madan [4], where it is shown that a European option with pay-off $f\left(S_{T}\right)$ ( $f$ sufficiently regular) can be hedged by a unique initial position of $f\left(S_{0}\right)-f^{\prime}\left(S_{0}\right) S_{0}$ unit discount bonds, $f^{\prime}\left(S_{0}\right)$ stock shares, and $f^{\prime \prime}(k) d k$ out-of-the-money call and put options of all strikes. Even more importantly, this result is model-free, so that it holds also in a continuous-time framework. For static hedging of exotic options in a diffusion setting, we refer to Carr et al. [3]. Moreover, in a discontinuous setting, Corcuera et al. [7] proved that a Lévy market can be completed by trading statically in power-jump assets, which are somewhat related to contracts on realized variance. In [6], a link between power-jump assets and European call options is established, allowing Corcuera and Guerra to prove that a Lévy market can be completed by trading in a continuum of European call options along strikes. Finally, in the paper [8], Davis and Obloj provide necessary and sufficient conditions for a market model to be complete by trading in given finite set of European-type derivatives driven by finitely many factors modelled as diffusion processes.

To sum up, all these papers deal with the problem of finding a good class of derivatives capable to span all contingent claims written on the same underlying. By "good class of derivatives" we mean a class of options sufficiently liquid in real financial markets in order to reproduce with a certain precision the hedging strategies suggested in these papers.

The present paper identifies American put options of all strike prices and with the same maturity as a good class of derivatives allowing an investor to replicate approximately (in $L^{2}$-sense) every positive contingent claim written on the same underlying in a very general frictionless financial market with one risk-free asset and one stock. Our main result, stated in Theorem 6, can be viewed as a generalization to a continuous-time setting of previously quoted Ross's result in [19] - notice that in Ross's single period setting there is no need to distinguish between European and path-dependent options like American puts. Moreover, our result is model-free. Indeed, we will make only the very mild assumption that the price process is a square-integrable and possibly discontinuous martingale with no jump at maturity. The price we have to pay is that the agent has to trade in infinitely many securities, in contrast with, e.g., Davis and Obloj [8] where, since agents can trade only in finitely many assets, the authors need to assume more regularity as, e.g., analiticity of the coefficients together with a non-singularity condition, to get the market completeness.

The present paper is structured as follows: In Section 2, we give a short description of the model, Section 3 contains the main result on hedging with American put options. Finally, in Section 4, we exhibit a two-periods market model where static investments in all European call (equivalently, put options) of all strike prices and all maturities are not enough for replicating all contingent claims, so justifying the use of their American counterparts.

\section{The model}

We consider a financial market composed by a riskless asset and a stock. More precisely, let $(\Omega, \mathcal{F}, \mathbb{P})$ be a probability space equipped with a filtration $\left(\mathcal{F}_{t}\right)_{t \in[0, T]}$, where $T>0$ is a finite horizon, $\mathcal{F}=\mathcal{F}_{T}$ and $\mathcal{F}_{0}$ is trivial. $\left(\mathcal{F}_{t}\right)_{t \in[0, T]}$ is the natural filtration - right-continuous and $\mathbb{P}$-saturated - generated 
by a strictly positive, càdlàg process $S=\left(S_{t}\right)_{t \in[0, T]}$ modelling the price of the risky asset. We assume without loss of generality that the price at time $t$ of the riskless asset satisfies $S_{t}^{0}=e^{r t}$ where $r>0$ is the spot interest rate (see Remark 7 for a straightforwad generalization). In the sequel $\mathbb{E}[\cdot]$ will denote expectation with respect to $\mathbb{P}$ and, for any process $X$, we set $\tilde{X}:=X / S^{0}$ and $\Delta X_{t}=X_{t}-X_{t-}$ for any $\left.\left.t \in\right] 0, T\right]$. Finally, $\mathcal{H}^{2}(\mathbb{P})$ will denote the space of all martingales bounded in $L^{2}(\mathbb{P}):=L^{2}\left(\mathbb{P}, \mathcal{F}_{T}\right)$.

Our first assumption concerns the behaviour of the risky asset process $S$.

Assumption $1 \tilde{S}=S / S^{0}$ belongs to $\mathcal{H}^{2}(\mathbb{P})$ and it does not jump at $T$, i.e. $\Delta S_{T}=0$ a.s..

Since by assumption $\left(\mathcal{F}_{t}\right)_{t \in[0, T]}$ is the natural filtration of $S$, the equality $\Delta S_{T}=0$ a.s. is equivalent to $\mathcal{F}_{T}=\mathcal{F}_{T-}$, i.e. the underlying filtration is left-continuous at $T$. We notice that this property is satisfied, e.g., by all diffusion models and also by all exponential Lévy models. Indeed, it is well-known that the natural filtration of any Lévy process is quasi-left continuous, i.e. it does not jump at predictable stopping times (see, e.g., Protter [17], p. 150, Exercises 8 and 9, and p. 191 for details).

In this setting, a contingent claim pay-off on $S$ with maturity $T$ is naturally modelled by a random variable $f \in L^{2}(\mathbb{P})$. This model is not necessarily complete, so there may exist infinitely many equivalent (local) martingale measures different from $\mathbb{P}$ and so, equivalently, not every contingent claim can be hedged by trading only in the underlying, so that allowing an investor to trade also in some class of options can enlarge considerably his hedging opportunities.

The following assumption can be viewed as a kind of no-arbitrage consistency among prices of different options written on the same underlying $S$.

Assumption 2 In this financial market every option price comes from the same equivalent martingale measure $\mathbb{P}$.

Observe that Assumption 2 has a very natural financial motivation. Indeed, let us forget about integrability issues for a while and consider two contingent claims with pay-offs $f$ and $f^{\prime}$ and corresponding risk-neutral prices $f_{t}$ and $f_{t}^{\prime}$ at time $t$. Assume that they come from two equivalent martingale measures $\mathbb{Q}$ and $\mathbb{Q}^{\prime}$ respectively, i.e. $f_{t}=\mathbb{E}_{\mathbb{Q}}\left[f \mid \mathcal{F}_{t}\right]$ and $f_{t}^{\prime}=\mathbb{E}_{\mathbb{Q}^{\prime}}\left[f^{\prime} \mid \mathcal{F}_{t}\right]$. If we want the enlarged market $\left(S, f, f^{\prime}\right)$ to be arbitrage-free, there must be an equivalent martingale measure $\mathbb{Q}^{\prime \prime}$ for $\left(S, f, f^{\prime}\right)$, so that one has

$$
f_{t}=\mathbb{E}_{\mathbb{Q}}\left[f \mid \mathcal{F}_{t}\right]=\mathbb{E}_{\mathbb{Q}^{\prime \prime}}\left[f \mid \mathcal{F}_{t}\right]
$$

and the same for $f^{\prime}$. This means that one can assume without loss of generality that the risk-neutral prices of $f$ and $f^{\prime}$ come from the same measure $\mathbb{Q}^{\prime \prime}$.

\section{Hedging with American put options}

In this section, we will prove that additional static investments in American put options of every strike price and with the same maturity $T$ allow an investor to hedge any contingent claim written on the underlying $S$. We make the following model assumption:

Assumption 3 American put options with any maturity $T$ and any strike price $k>0$ are available for trading and all are issued at $t=0$.

Let us denote $\mathcal{T}$ the set of all $[0, T]$-valued stopping times and $\widehat{\tau}_{k}$ the smallest optimal exercise time corresponding to a given strike price $k$. Such stopping times do exist (see Remark 4 for details) so that we have

$$
P_{0}\left(k, \widehat{\tau}_{k}\right):=\sup _{\tau \in \mathcal{T}} \mathbb{E}\left[e^{-r \tau}\left(k-S_{\tau}\right)^{+}\right]=\mathbb{E}\left[e^{-r \widehat{\tau}_{k}}\left(k-S_{\widehat{\tau}_{k}}\right)^{+}\right],
$$


which is the price that an agent must pay for buying at time 0 an American put option with strike $k$ and maturity $T$. More generally, we set

$$
\tilde{P}_{t}(k, \tau):=\mathbb{E}\left[e^{-r \tau}\left(k-S_{\tau}\right)^{+} \mid \mathcal{F}_{t}\right],
$$

for any stopping time $\tau \in \mathcal{T}$. Notice that under our assumptions each price process $\tilde{P}(k, \tau)$ is a martingale in $\mathcal{H}^{2}(\mathbb{P})$.

Remark 4 Observe that $\widehat{\tau}_{k}$ exists for all strike prices $k>0$. Indeed, the underlying discounted price process $\tilde{S}$ is a strictly positive martingale in $\mathcal{H}^{2}(\mathbb{P})$ over a finite time interval $[0, T]$, so that it is of class $(D)$ and, obviously, constant in expectations. Thus, Théorème 2.43 in El Karoui St. Flour's Lecture Notes [9] applies, so giving existence of $\widehat{\tau}_{k}$ for all $k>0$.

Now, we consider the set $\mathcal{R}_{a}$ of all discounted contingent claims which can be approximately replicated by investing dynamically in the underlying $S$ and statically in finitely many American put options as follows: the American puts that the agent buys at time $t=0$ can be exercised at any stopping time $\tau \in \mathcal{T}$, while the American puts that he sells will be exercised at their corresponding optimal times by their buyers.

Mathematically speaking, $\mathcal{R}_{a}$ is the set of all $\mathcal{F}_{T}$-measurable random variables of the form

$$
x+\int_{0}^{T} \theta_{t} d \tilde{S}_{t}+\sum_{i=1}^{n} \alpha_{i}\left(\tilde{P}_{T}\left(k_{i}, \tau_{i}\right)-P_{0}\left(k_{i}, \widehat{\tau}_{k_{i}}\right)\right)-\sum_{j=1}^{m} \beta_{n+j}\left(\tilde{P}_{T}\left(k_{n+j}, \widehat{\tau}_{k_{n+j}}\right)-P_{0}\left(k_{n+j}, \widehat{\tau}_{k_{n+j}}\right)\right)
$$

where

- $x \in \mathbb{R}$ is the initial endowment of the agent,

- $\theta$ is a real-valued $S$-integrable predictable process such that $\int \theta d \tilde{S}$ is a martingale in $\mathcal{H}^{2}(\mathbb{P})$ modelling the dynamic investment strategy in the stock $S$,

- $n \geq 0$ is the number of American puts that the agent buys at time 0 , while $m \geq 0$ is the number of American puts sold at time 0,

- each weight $\alpha_{i} \geq 0$ is a nonnegative real number representing the number of American puts with strike $k_{i}(i=1, \ldots, n)$ bought by the agent at time 0 paying the price $P_{0}\left(k_{i}, \widehat{\tau}_{k_{i}}\right)$, while $\beta_{n+j} \geq 0(j=1, \ldots, m)$ represents the number of American puts with strike $k_{n+j}$ sold by the agent at time 0 receiving the price $P_{0}\left(k_{n+j}, \widehat{\tau}_{k_{n+j}}\right)$.

We adopt the convention that any summation over an empty set of indexes is equal to zero. Notice that $\mathcal{R}_{a}$ is not a linear subspace, it is nonetheless a convex cone. We will denote $\mathcal{R}_{a}^{*}$ its (positive) dual, i.e.

$$
\mathcal{R}_{a}^{*}:=\left\{f \in L^{2}(\mathbb{P}): \mathbb{E}[f g] \geq 0, \forall g \in \mathcal{R}_{a}\right\},
$$

and by $\mathcal{R}_{a}^{* *}$ its bidual, i.e. $\mathcal{R}_{a}^{*}:=\left(\mathcal{R}_{a}^{*}\right)^{*}$. We recall that the bidual $C^{* *}$ of any convex cone $C$ in a vector topological space coincides with the closure of $C$, and that for any pair of convex cones $C$ and $C^{\prime}$ such that $C \subset C^{\prime}$ one has $C^{*} \supset\left(C^{\prime}\right)^{*}$ (see, e.g, [22], Chapter 1).

Remark 5 Notice that in the first summation appearing in (2), denoting the final gain coming from a long position taken at time $t=0$ in American puts with strikes $k_{i}$, the puts are not necessarily exercised at their optimal exercise times $\widehat{\tau}_{k_{i}}$. The agent, willing to hedge against the risk of a given final pay-off $f$, can in principle exercise his puts at any stopping time between today and the maturity $T$. The question if hedging purposes may lead an agent to exercise such options at sub-obtimal times remains open. 
Our main result is that $\mathcal{R}_{a} \cap L_{+}^{2}(\mathbb{P})$ is dense in $L_{+}^{2}(\mathbb{P})$ which denotes the set of all positive random variables in $L^{2}(\mathbb{P})$. In financial terms, it means that every positive contingent claim can be (approximately) replicated by a mixed investment as in (2): dynamic in the underlying and static in American put options.

Theorem 6 Under our assumptions, the closure of $\mathcal{R}_{a}$ contains $L_{+}^{2}(\mathbb{P})$.

Proof. We recall once more that the closure of a convex cone $C$ in a topological vector space equals its bidual $C^{* *}$, so that all we need to prove is that the bidual $\mathcal{R}_{a}^{* *}$ contains $L_{+}^{2}(\mathbb{P})$. In order to do that, it suffices to show that $L_{+}^{2}(\mathbb{P})$ contains the dual cone $\mathcal{R}_{a}^{*}$, i.e. that any random variable $N_{T} \in L^{2}(\mathbb{P})$ which is positive over $\mathcal{R}_{a}$, i.e. $\mathbb{E}\left[N_{T} f\right] \geq 0$ for all $f \in \mathcal{R}_{a}$, is a.s. positive itself. Denote $N$ the martingale in $\mathcal{H}^{2}(\mathbb{P})$ associated to $N_{T}$, i.e. $N$ is the càdlàg version of the martingale $\mathbb{E}\left[N_{T} \mid \mathcal{F}_{t}\right]$, for $t \in[0, T]$.

First observe that, since $\mathcal{R}_{a}$ contains $\mathbb{R}$, we have $\mathbb{E}\left[N_{T}\right]=N_{0}=0$. From the fact that $\mathbb{E}\left[N_{T} f\right] \geq 0$ for any $f$ equal to the static hedging part appearing in (2), we can deduce in particular that

$$
\alpha_{1} \mathbb{E}\left[N_{T} \tilde{P}_{T}\left(k_{1}, \tau_{1}\right)\right]-\alpha_{2} \mathbb{E}\left[N_{T} \tilde{P}_{T}\left(k_{2}, \widehat{\tau}_{k_{2}}\right)\right] \geq 0
$$

for all nonnegative real numbers $\alpha_{1}, \alpha_{2}$, all strikes $k_{1}, k_{2}$, and all stopping times $\tau_{1} \in \mathcal{T}$. Taking $\alpha_{2}=0$, we get that

$$
0 \leq \mathbb{E}\left[N_{T} \tilde{P}_{T}\left(k_{1}, \tau_{1}\right)\right]=\mathbb{E}\left[N_{T} \tilde{P}_{\tau_{1}}\left(k_{1}, \tau_{1}\right)\right]=\mathbb{E}\left[N_{\tau_{1}} e^{-r \tau_{1}}\left(k_{1}-S_{\tau_{1}}\right)^{+}\right]=\mathbb{E}\left[\tilde{N}_{\tau_{1}}\left(k_{1}-S_{\tau_{1}}\right)^{+}\right],
$$

for every strike price $k_{1}$ and every stopping time $\tau_{1} \in \mathcal{T}$. Doob's optional sampling theorem implies that the process $\tilde{N}(k-S)^{+}$is a $\mathbb{P}$-submartingale for every $k>0$. The integration by parts formula gives

$$
\tilde{N}_{t}\left(k-S_{t}\right)^{+}=\tilde{N}_{t}\left(S_{t}-k\right)^{-}=\int_{0}^{t}\left(S_{u-}-k\right)^{-} d \tilde{N}_{u}+\int_{0}^{t} \tilde{N}_{u-} d(S-k)_{u}^{-}+\left[\tilde{N},(S-k)^{-}\right]_{t}
$$

for all $t \in[0, T]$. By Tanaka's formula for discontinuous semimartingales (e.g. Protter [17], Theorem 68, p. 216) and since $d S_{u}=r S_{u} d u+e^{r u} d \tilde{S}_{u}$, we have that

$$
\begin{aligned}
\int_{0}^{t} \tilde{N}_{u-} d(S-k)_{u}^{-}= & -\int_{0}^{t} \tilde{N}_{u-} \mathbf{1}_{\left\{S_{u-} \leq k\right\}}\left(r S_{u} d u+e^{r u} d \tilde{S}_{u}\right) \\
& +\sum_{0<u \leq t} \tilde{N}_{u-}\left[\mathbf{1}_{\left\{S_{u-}>k\right\}}\left(S_{u}-k\right)^{-}+\mathbf{1}_{\left\{S_{u-} \leq k\right\}}\left(S_{u}-k\right)^{+}\right] \\
& +\frac{1}{2} \int_{0}^{t} \tilde{N}_{u-} d L_{u}^{k}(S),
\end{aligned}
$$

for every instant $t \in[0, T]$ and strike price $k>0$.

The fact that $N_{T}$ belongs to the dual of $\mathcal{R}_{a}$ implies also that $N_{T}$ is weakly orthogonal to $\tilde{S}$ and so strongly orthogonal to $\widetilde{S}$ as well, ${ }^{1}$ i.e. $N \tilde{S}=\tilde{N} S$ belongs to $\mathcal{H}^{1}(\mathbb{P})$, the space of all martingales bounded in $L^{1}(\mathbb{P})$ (use, e.g., Lemma 2, Section IV, in [17]). Moreover, being $d\left[\tilde{N},(S-k)^{-}\right]_{t}=$ $e^{-r t} d\left[N,(S-k)^{-}\right]_{t}$ and $L^{k}(S)$ a continuous increasing process, one has

$$
\left[\tilde{N},(S-k)^{-}\right]_{t}=\sum_{0<u \leq t} \Delta \tilde{N}_{u} \Delta(S-k)_{u}^{-},
$$

\footnotetext{
${ }^{1}$ We recall that, in this $\mathcal{H}^{2}$ setting, weak orthogonality between two martingales $M$ and $N$ in $\mathcal{H}^{2}(\mathbb{P})$ is equivalent to strong orthogonality, i.e. $M N \in \mathcal{H}^{1}(\mathbb{P})$. See, e.g., Lemma 2, Section IV, in Protter's book [17].
} 
which is a pure jump process. Notice that $\tilde{N}(k-S)^{+}$is a $\mathbb{P}$-submartingale with Doob-Meyer decomposition $\tilde{N}(k-S)^{+}=M+B$, where $M$ is a local martingale and $B$ is a predictable increasing process. The local martingale part $M$ include certainly the following term

$$
\int_{0}^{t}\left(S_{u-}-k\right)^{-} d \tilde{N}_{u}-\int_{0}^{t} \tilde{N}_{u-} \mathbf{1}_{\left\{S_{u-} \leq k\right\}} e^{r u} d \tilde{S}_{u}
$$

and, since any càdlàg local martingale with finite variation must be purely discontinuous (see, e.g., [15, Lemma $4.14 \mathrm{~b})]$ ),$M$ cannot contain the finite variation terms with continuous paths appearing in (3) and (4). Thus, those terms have to belong to the increasing part $B$, which could in principle contains some additional terms of pure jump type. An important consequence of it is that the process

$$
\frac{1}{2} \int_{0}^{t} \tilde{N}_{u-} d L_{u}^{k}(S)-\int_{0}^{t} r k \tilde{N}_{u} \mathbf{1}_{\left\{S_{u} \leq k\right\}} d u, \quad t \in[0, T],
$$

must be increasing, i.e.

$$
\int_{s}^{t} r k \tilde{N}_{u} \mathbf{1}_{\left\{S_{u} \leq k\right\}} d u \leq \frac{1}{2} \int_{s}^{t} \tilde{N}_{u-} d L_{u}^{k}(S),
$$

for all $s, t \in[0, T]$ with $s \leq t$ and all $k>0$. Using a standard class monotone argument, the inequality in (5) can be generalized as follows

$$
\int_{A} r k \tilde{N}_{u} \mathbf{1}_{\left\{S_{u} \leq k\right\}} d u \leq \frac{1}{2} \int_{A} \tilde{N}_{u-} d L_{u}^{k}(S),
$$

for all Borel set $A$ and all $k>0$. First, observe that in the two integrals in (6) the same function $u \rightarrow \tilde{N}_{u-}$ is integrated with respect to two $d t$-a.e. mutually singular ${ }^{2}$ measures $(1 / 2) d L_{u}^{k}(S)$ and $r k \mathbf{1}_{\left\{S_{u} \leq k\right\}} d u$, which implies that such an inequality is verified only if $N \leq 0 d \mathbb{P} \otimes d t$-a.e. on the set $\left\{(\omega, t): S_{t}(\omega) \leq k\right\}$, for all $k>0$. As a consequence, one has $N \leq 0 d \mathbb{P} \otimes d t$-a.e. on $\Omega \times[0, T]$ and, since $N$ is càdlàg, one has also that $N_{T-} \leq 0$ a.s.. Finally, notice that Assumption 1 consequence $\mathcal{F}_{T}=\mathcal{F}_{T-}$ implies that no martingale can jump at $T$ (see Protter [17], p. 191, for details), so that one has also that $N_{T} \leq 0$ a.s.. To end the proof, it suffices to recall that $N$ is martingale with $N_{0}=0$, so that $\mathbb{E}\left[N_{T}\right]=0$ and, $N_{T}$ being a.s. negative, it must be a.s. zero.

Remark 7 A careful inspection of our proof reveals that Theorem 6 holds true even if the spot interest rate $r$ is not necessarily constant but a positive and bounded deterministic function of time, more precisely: $S_{t}^{0}=\exp \left(\int_{0}^{t} r(u) d u\right)$ where $r(u)$ is measurable positive function defined on $[0, T]$ and such that $S_{T}^{0}$ is bounded above by some constant.

Remark 8 Note that if one considers contingent claims depending on some randomness source different from $S$, then the previous completeness result breaks down. Indeed, just consider a model whose price processes are identically equal to one, i.e. $S^{0} \equiv S \equiv 1$, so that the natural filtration of $S$ is trivial and the collection of all American put option pay-offs $\left\{(k-1)^{+}: k>0\right\}$ coincides with $[0, \infty)$. Thus, $\mathcal{R}_{a}=\mathbb{R}$. Then, consider a sufficiently large filtration $\left(\mathcal{F}_{t}\right)_{t \in[0, T]}$ such that $\mathcal{F}_{T}$ contains at least one non-degenerate square-integrable positive random variable $f$. It is now clear that, even if we allow the strategies to be $\mathbb{F}$-adapted, in such a market it is not possible to hedge $f$ as in Theorem 6.

\footnotetext{
${ }^{2}$ Indeed, the support of $d L_{u}^{k}(S)$ is $\left\{u: S_{u}=S_{u-}=k\right\}$ (see, e.g., Protter's book [17], Theorem 69, p. 217) while that of $\mathbf{1}_{\left\{S_{u} \leq k\right\}} d u$ is $\left\{u: S_{u} \leq k\right\}$. Thus, their intersection is contained in $\left\{u: S_{u}=k\right\}$ which is at most countable and so it has zero Lebesgue measure.
} 


\section{A counter-example to hedging with European call options}

In this section, we will exhibit a financial market in discrete time with a finite horizon $T \in \mathbb{N}$ and defined on a finite probability space, where it is not possible to hedge all contingent claims by trading dynamically in a given underlying and statically in all European call options of every strike price $k>0$ and every maturity before $T$.

Let $(\Omega, \mathcal{F}, \mathbb{P})$ be a finite probability space supporting a martingale $S=\left(S_{t}\right)_{t=0}^{T}$ modelling the price evolution of a stock. This space is assumed to be equipped with the filtration $\left(\mathcal{F}_{t}\right)_{t=0}^{T}$ naturally generated by $S$ and for which $\mathcal{F}=\mathcal{F}_{T}$. As usual, we denote $S^{0}$ the price process for a riskless asset and assume $S^{0} \equiv 1$. For a given discrete-time process $X$, we set $\Delta X_{t}:=X_{t}-X_{t-1}, t=1, \ldots, T$.

Consider the linear space $\mathcal{R}_{e}$ spanned by all random variables $f$ of the form

$$
f=x+\sum_{t=1}^{T} \theta_{t} \Delta S_{t}+\sum_{i=1}^{n} \alpha_{i}\left(C_{T}\left(T_{i}, k_{i}\right)-C_{0}\left(T_{i}, k_{i}\right)\right),
$$

where $x \in \mathbb{R}$ is an initial endowment, $\theta$ is any predictable process modelling the dynamic strategy in $S, \alpha=\left(\alpha_{1}, \ldots, \alpha_{n}\right) \in \mathbb{R}^{n}$ is any static strategy in $n \geq 1$ European call options with maturities $T_{i} \leq T$ and strike prices $k_{i}$ for $1 \leq i \leq n$, whose no-arbitrage prices are denoted by $C_{t}\left(T_{i}, k_{i}\right):=$ $\mathbb{E}\left[\left(S_{T_{i}}-k_{i}\right)^{+} \mid \mathcal{F}_{t}\right] . n \geq 1$ is an arbitrary positive integer.

Our aim is to construct a process $S$ such that $\mathcal{R}_{e}$ is not dense in the set of all positive $\mathcal{F}_{T^{-}}$ measurable random variables $L_{+}^{0}$, equipped with the usual scalar product $(f, g)=\mathbb{E}[f g]$. To do so, we use the following consequence of Theorem 3 in Campi [1]. We provide its short proof for reader's convenience.

Lemma 9 Assume that $\mathcal{R}_{e} \cap L_{+}^{0}$ is dense in $L_{+}^{0}$. Then the set of all $\mathbb{P}$-equivalent martingale measures $\mathbb{Q}$ under which $S$ has the same marginals as under $\mathbb{P}$ reduces to a singleton.

Proof. Let $\mathbb{Q}$ be an equivalent martingale measure under which $S$ has same marginals as under $\mathbb{P}$. In this case, for all postive random variable $f$ as in $(7)$ we have $\mathbb{E}[f]=x=\mathbb{E}_{\mathbb{Q}}[f]$ and, since the family of those random variables is assumed to be dense in $L_{+}^{0}$, we can conclude that $\mathbb{Q}=\mathbb{P}$ on $\mathcal{F}_{T}$.

In the light of that result, it suffices now to exhibit a process $S$ admitting two different equivalent martingale measures $\mathbb{P}$ and $\mathbb{Q}$ under which $S$ has the same marginals. Here it is: $T=2, S_{0}=3 / 2$, the marginals at time $t=1$ are given by $\mathbb{P}\left[S_{1}=1\right]=\mathbb{Q}\left[S_{1}=1\right]=1 / 2$ and $\mathbb{P}\left[S_{1}=2\right]=\mathbb{Q}\left[S_{1}=2\right]=1 / 2$, and $S_{2}$ takes the values $0,1,2,3$ each one with probability $1 / 4$ under both $\mathbb{P}$ and $\mathbb{Q}$. To complete the description of $\mathbb{P}$ and $\mathbb{Q}$, we only need to assign the transition probabilities between $t=1$ and $t=2$. This can be done in many ways to get $\mathbb{P}, \mathbb{Q} \in \mathcal{M}$ and nonetheless keep them different. For instance, set $p_{i j}:=\mathbb{P}\left[S_{2}=j \mid S_{1}=i\right]$ and $q_{i j}:=\mathbb{Q}\left[S_{2}=j \mid S_{1}=i\right]$ for $i, j \in\{0,1,2,3\}$, and consider $p_{23}=p_{10}=0.4, p_{22}=p_{11}=0.3, p_{21}=p_{12}=0.2$ and $p_{20}=p_{13}=0.1$ for the measure $\mathbb{P}$, and $q_{23}=q_{10}=0.39, q_{22}=q_{11}=0.33, q_{21}=q_{12}=0.17$ and $q_{20}=q_{13}=0.11$ for the measure $\mathbb{Q}$. It can be easily verified that this example is exactly what we were looking for.

Remark 10 As it is formulated, this example does not satisfy the assumption $S_{T}=S_{T-\text {. Indeed, }}$ $S_{T-}=S_{2-}=S_{1} \neq S_{2}$. Nonetheless, it can be easily embedded in the framework of the previous section, where the price process $S$ is assumed to be left-continuous at $T$ as in Assumption 1, by simply adding a date $T+1$ and setting $S_{T+1}=S_{T}$.

Remark 11 By the call-put parity, trading in all European call options as in (7) is equivalent to trading in $S$ and in all European put options. As a consequence, our example also shows that it is in general not possible to replicate each square-integrable positive contingent claim by trading dynamically in the underlying and statically in all European put options. 


\section{References}

[1] Campi, L.: Arbitrage and completeness in financial markets with given $N$-dimensional distributions. Decis. Econ. Finance 27 (2004), no. 1, 57-80.

[2] Carmona, R., Nadtochiy, S.: Local volatility dynamic models. Finance and Stochastics, 13 (2009), Issue 1, 1-48.

[3] Carr, P., Ellis, K., Gupta, V.: Static Hedging of Exotic Options. The Journal of Finance 53 (1998), Issue 3, 1165-1190.

[4] Carr, P., Madan, D.B.: Optimal positioning in derivative securities. Quantitative Finance 1 (2001), 19-37.

[5] Carr, P., Jin, X., Madan, D.B.: Optimal investment in derivative securities. Finance Stoch. 5 (2001), no. 1, 33-59.

[6] Corcuera, J.M., Guerra, J.: Dynamic complex hedging in additive markets. Preprint, IMUB, Universitat de Barcelona, 2007.

[7] Corcuera, J. M., Nualart, D., Schoutens, W.: Completion of a Lévy market by power-jump assets. Finance Stoch. 9 (2005), no. 1, 109-127.

[8] Davis, M., Obloj, J.: Market completion using options, in Advances in Mathematics of Finance, ed. L. Stettner, Banach Center Publications vol. 43 (2008) 49-60, Polish Academy of Sciences, Warsaw.

[9] El Karoui, N.: Les aspects probabilistes du contrôle stochastique. (French) [The probabilistic aspects of stochastic control] Ninth Saint Flour Probability Summer School 1979, pp. 73-238. Lecture Notes in Math. 876, Springer, Berlin-New York, 1981.

[10] Green, R. C., Jarrow, R. A.: Spanning and completeness in markets with contingent claims. $J$. Econom. Theory 41 (1987), no. 1, 202-210.

[11] Ilhan, A., Sircar, R.: Optimal static-dynamic hedges for barrier options. Math. Finance 16 (2006), no. 2, 359-385.

[12] Ilhan, A., Jonsson, M., Sircar, R.: Optimal investment with derivative securities. Finance Stoch. 9 (2005), no. $4,585-595$.

[13] Jacka, S. D.: A martingale representation result and an application to incomplete financial markets. Math. Finance 2, 23-34 (1992)

[14] Jacod, J., Protter, Ph.: Risk Neutral Compatibility with Option Prices. Finance and Stochastics, 14 (2010), no. 2, 285-315.

[15] Jacod, J., Shiryaev, A.N. (2003): Limit Theorems for Stochastic Processes. Springer, Berlin, Germany.

[16] Nachman, D.: Spanning and Completeness with Options. The Review of Financial Studies Fall 1988.

[17] Protter, Ph.: Stochastic integration and differential equations. Second edition. Version 2.1. Corrected third printing. Stochastic Modelling and Applied Probability, 21. Springer-Verlag, Berlin, 2005. 
[18] Rogge, L.: Call completeness implies completeness in the $n$-period model of a financial market. Finance Stoch. 10 (2006), no. 2, 298-301.

[19] Ross, S.: Options and efficiency. Q. J. Econ. 90 (1976).

[20] Schweizer, M., Wissel,J.: Term structures of implied volatilities: Absence of arbitrage and existence results. Mathematical Finance, 18 (2008), no. 1, 77-114.

[21] Schweizer, M., Wissel, J.: Arbitrage-free market models for option prices: The multi-strike case. Finance and Stochastics, 12 (2008), no. 4, 469-505.

[22] Zălinescu, C.: Convex analysis in general vector spaces, World Scientific, New Jersey, 2002. 